\title{
Cell-Assisted Lipotransfer for Cosmetic Breast Augmentation: Supportive Use of Adipose-Derived Stem/Stromal Cells
}

\author{
Kotaro Yoshimura $\cdot$ Katsujiro Sato $\cdot$ Noriyuki Aoi $\cdot$ Masakazu Kurita $\cdot$ \\ Toshitsugu Hirohi · Kiyonori Harii
}

Received: 27 April 2007/Accepted: 22 May 2007/Published online: 1 September 2007

(C) Springer Science+Business Media, LLC 2007

\begin{abstract}
Background Lipoinjection is a promising treatment but has some problems, such as unpredictability and a low rate of graft survival due to partial necrosis.

Methods To overcome the problems with lipoinjection, the authors developed a novel strategy known as cellassisted lipotransfer (CAL). In CAL, autologous adiposederived stem (stromal) cells (ASCs) are used in combination with lipoinjection. A stromal vascular fraction (SVF) containing ASCs is freshly isolated from half of the aspirated fat and recombined with the other half. This process converts relatively ASC-poor aspirated fat to ASC-rich fat. This report presents the findings for 40 patients who underwent CAL for cosmetic breast augmentation.
\end{abstract}

Partially presented at the 3rd annual meeting of the International Fat Applied Technology Society (IFATS), Charlottesville, Virginia, 11 September 2005, and the 8th annual meeting of Tissue Engineering Society International (TESI), Shanghai, China, 23 October 2005.

K. Yoshimura $(\bowtie) \cdot$ N. Aoi

Department of Plastic Surgery, University of Tokyo School of Medicine, 7-3-1 Hongo, Bunkyo-ku, 113-8655 Tokyo, Japan

e-mail: yoshimura-pla@h.u-tokyo.ac.jp

K. Sato

Cellport Clinic Yokohama, Yokohama Excellent III Building 2F, 3-35, Minami-nakadori, Naka-ku, Yokohama, 231-0006

Kanagawa, Japan

M. Kurita $\cdot$ K. Harii

Department of Plastic Surgery, Kyorin University School of Medicine, 6-20-2 Shinkawa, Mitaka-shi, 181-8611 Tokyo, Japan

T. Hirohi

Ritz Cosmetic Surgery Clinic Tokyo, Meguro Toho Building 8F,

3-1-7, Kamiosaki, Shinagawa-ku, 141-0021 Tokyo, Japan
Results Final breast volume showed augmentation by 100 to $200 \mathrm{ml}$ after a mean fat amount of $270 \mathrm{ml}$ was injected. Postoperative atrophy of injected fat was minimal and did not change substantially after 2 months. Cyst formation or microcalcification was detected in four patients. Almost all the patients were satisfied with the soft and naturalappearing augmentation.

Conclusions The preliminary results suggest that CAL is effective and safe for soft tissue augmentation and superior to conventional lipoinjection. Additional study is necessary to evaluate the efficacy of this technique further.

Keywords Adipose-derived stem/stromal cells . Breast augmentation - Cell therapy · Lipoinjection · Tissue engineering

Autologous fat transplantation is one promising treatment for facial rejuvenation and soft tissue augmentation because it results in no incisional scar or complications associated with foreign materials. However, certain problems remain, such as unpredictability and a low rate of graft survival due to partial necrosis. Many innovations to overcome these problems have been reported [1, 2, 4-6, 18] and reviewed previously [4, 14]. On the basis of these reports, we tentatively concluded that we could harvest fat with a $2.5-\mathrm{mm}$ cannula or 18-gauge needle at a vacuum lower than $700 \mathrm{mmHg}$ and reinject it using an 18-gauge needle without significant adipocyte damage [14].

Lipoinjection can be used to treat facial changes associated with aging and to correct various types of depressed deformities such as hemifacial microsomia and pectus excavatum. It also has been used in breast augmentation by a limited number of plastic surgeons [3], although the use of autologous fat for breast augmentation has been 
controversial. Consensus is lacking on whether lipoinjection is safe and appropriate because of microcalcifications that may cause confusion in the evaluation of mammograms. Recently, autologous fat injection has been reevaluated as a potential alternative to artificial implants for breast augmentation [3, 15, 16, 19]. This reevaluation may reflect recent advances in autologous fat transfer and the radiologic detection of breast cancer.

To overcome the problems associated with autologous fat transfer, we use a novel strategy known as cell-assisted lipotransfer (CAL) (Fig. 1). Findings have shown that tissue-specific progenitor cells in the adipose tissue have the capacity to differentiate into various cell lineages [21]. Thus, the progenitors, currently known as adipose-derived stem/stromal cells (ASCs), are expected to become a valuable tool in a wide range of cell-based therapies.

The therapeutic concept of CAL was described in our previous report on preclinical studies [9]. We found that aspirated fat has approximately half the number of ASCs found in excised whole fat. There are two main reasons for this relative deficiency. First, a major portion of the ASCs is located around large vessels and left in the donor site after liposuction [9]. Second, a part of the ASCs is released into the fluid portion of liposuction aspirates [20]. The relative deficiency of ASCs may induce postoperative long-term atrophy of injected fat, as partially confirmed in animal studies $[8,9,11]$.

With the CAL strategy, autologous ASCs are used to enhance angiogenesis, to improve the survival rate of grafts, and to reduce postoperative atrophy. In CAL, half the volume of the aspirated fat is processed for isolation of the stromal vascular fraction (SVF) containing ASCs. During the isolation process, the other half of the aspirated fat is prepared for grafting. Freshly isolated SVF, which we characterized previously [20], is attached to the aspirated fat, with the fat acting as a living scaffold before transplantation. Finally, the SVF-supplemented fat is injected into the target sites. Thus, ASC-poor fat is converted to ASC-rich fat in the preparation process of the injectable material.

In this report, we describe the preliminary results experienced by patients who underwent CAL for cosmetic breast augmentation. This is the first report on the clinical use of ASCs for cosmetic purposes.

\section{Materials and Methods}

Patients

From 2003 to 2007, we performed CAL for 70 patients: in the breast for 60 patients (including 8 patients who had breast reconstruction after mastectomy), in the face for 12 patients, and in the hip for 1 patient. For three patients, CAL was performed at two sites. Informed consent was obtained from all the patients. The study protocol conformed to the guidelines of the 1975 Declaration of Helsinki and was approved by individual institutional review boards.

In this study, 40 patients with healthy thoraxes and breasts underwent CAL for purely cosmetic breast augmentation. Patients undergoing breast reconstruction for inborn anomaly or after mastectomy were not included. At this writing, 19 of these 40 patients have been followed for more than 6 months, and the maximum follow-up period has been 42 months. All the patients were Japanese women with a mean body mass index (BMI) of $19.1 \pm 1.9$. Their ages varied from 20 to 62 years (mean, $35.8 \pm 9.1$ ). The
Fig. 1 Scheme of cell-assisted lipotransfer. Relatively adiposederived stem/stromal cell (ASC)-poor aspirated fat is converted to ASC-rich fat by supplementing ASCs isolated from the other half of the aspirated fat. The ASCs are attached to the aspirated fat, which is used as a scaffold in this strategy

\section{Cell-Assisted Lipotransfer (CAL)}

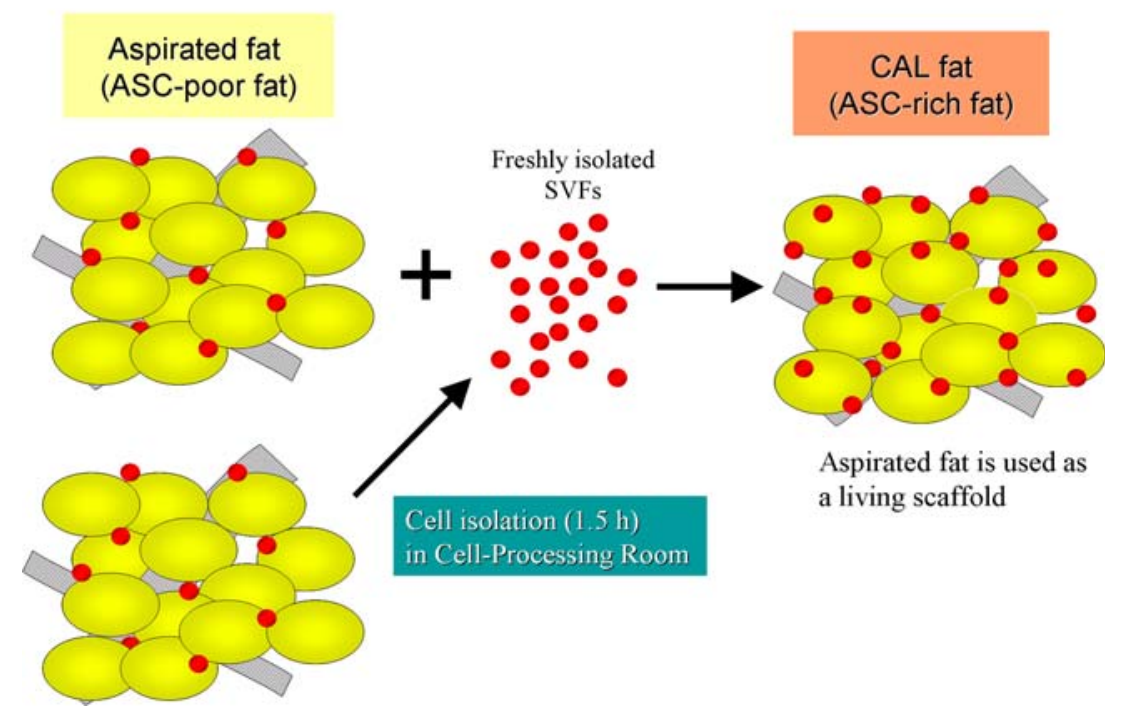


Table 1 Patient data

\begin{tabular}{ll}
\hline No. of cases & 40 \\
\hline Sex & $40 \mathrm{~F}, 0 \mathrm{M}$ \\
Age (years) & $35.8 \pm 9.1$ \\
BMI & $19.7 \pm 1.9$ \\
Surgical procedure & \\
Group A & 6 \\
Group B & 2 \\
Group C & 32 \\
Site of liposuction & \\
Thighs & 25 \\
Thighs and abdomen & 13 \\
Thighs and lower legs & 2 \\
Total volume of suctioned fat (ml) & $1111.8 \pm 164.0$ \\
Volume of injection (ml) & \\
Left & $268.1 \pm 47.6$ \\
Right & $277.3 \pm 39.1$ \\
Operation time (min) & $257.1 \pm 39.1$ \\
\hline
\end{tabular}

mean volume of injected fat was $268.1 \pm 47.6 \mathrm{ml}$ on the left side and $277.3 \pm 39.1 \mathrm{ml}$ on the right side. The demographic and surgical data for these patients are summarized in Table 1.

\section{Surgical Techniques}

Before the procedure began, the liposuction site was infiltrated with combined saline solution and diluted epinephrine $(0.001 \%)$. With the patient under general anesthesia, adipose tissue was suctioned using a cannula with a $2.5-\mathrm{mm}$ inner diameter and a conventional liposuction machine. Approximately half of the collected liposuction aspirate was used for isolation of the SVF from both the adipose and fluid portions of the liposuction aspirates, as described previously [20]. This cell processing procedure required about $90 \mathrm{~min}$. During the processing period, the other half of the lipoaspirate was harvested as graft material.

The adipose portion of the liposuction aspirates was either washed several times and placed in an upright position to obtain clear separation of fluids and oil (groups $\mathrm{A}$ and $\mathrm{B}$ ) or centrifuged at $700 \mathrm{~g}$ for $3 \mathrm{~min}$ without washing (group C), then put into a metal jar $(500 \mathrm{ml})$, which was placed in water with crushed ice. In groups A and C, the fresh SVF isolated from both the adipose and fluid portions was added to the graft material. After gentle mixing and a wait of 10 to $15 \mathrm{~min}$ for cell adherence to the aspirated fat, the cell-supplemented fat was put into an injection syringe. In Group B, the freshly isolated SVF was resuspended in
$60 \mathrm{ml}$ of saline, then diffusely injected into the whole breast mounds separately ( $30 \mathrm{ml}$ for each breast) immediately after conventional lipoinjection. There were 6 patients in group A, 2 patients in group B, and 32 patients in group $\mathrm{C}$.

For the injection syringe, a 10-ml LeVeen inflator (Boston Scientific Corp., Boston, MA, USA) or our original 20-ml syringe was used because each is a screw-type syringe (with a threaded plunger), and the threaded connections fit both the connecting tube and the needle to allow precise control during injection. To reduce the time of the procedure, two syringes were used. While the one syringe was being used for an injection, the other was being filled with the graft material in preparation for the next injection. An 18-gauge needle (150 mm long) was used for lipoinjection and inserted subcutaneously at one of four points indicated in Figs. 2A and B.

The operator took care to insert and place the needle horizontally (parallel to the body) to avoid damaging the pleura and causing a pneumothorax. The needle was inserted in several layers and directions. It was continuously and gradually retracted as the plunger was advanced. This technique was used to obtain a diffuse distribution of the graft material (Figs. 2 and 3). The grafts were injected into the fatty layers on, around, and under the mammary glands, and also into the pectoralis muscles.

\section{Results}

The transplantation of adipose tissue was successfully performed in all cases, and the time of the injection process ranged from 35 to $60 \mathrm{~min}$ for both breasts. Subcutaneous bleeding, occasionally seen in some parts of the breasts, resolved in 1 to 2 weeks.

Transplanted adipose tissue was gradually absorbed during the first 2 postoperative months (especially during the first month), and the breast volume showed a minimal change thereafter, although skin tension sometimes lessened after 2 months. Three representative surgical sites are shown in Figs. 4 to 9. The difference in breast circumference (chest circumference at the nipple minus the chest circumference at the inframammary fold) had increased in all cases by 4 to $8 \mathrm{~cm}$ at 6 months, which corresponds to two to three brassiere cup sizes. This circumference increase appeared to correspond to the 100- to 200-ml increase in the volume of each breast mound, which was partially confirmed by our preliminary evaluation using a three-dimensional quantitative measurement system.

Compared with breast augmentation using implants of the same size, augmentation with CAL showed a lower height but a more natural contour of the breasts. All cases but one (see later) showed natural softness of the breasts 

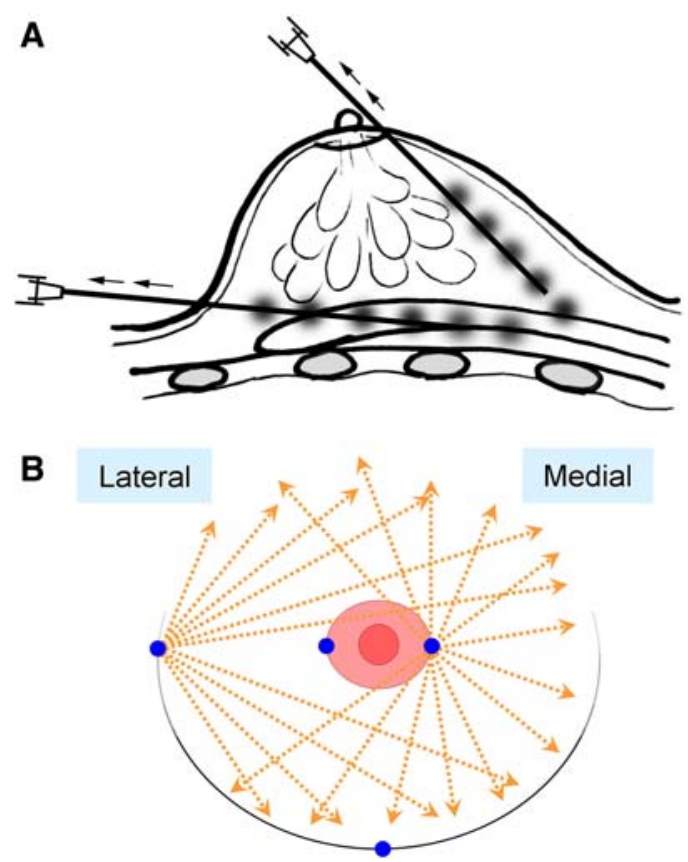

Fig. 2 Schematic instruction of the injection method. (A) A small amount of fat tissue is injected as small aliquots or as a thin string with a long needle, using a syringe with a threaded plunger, while the needle is continuously withdrawn. (B) The needle is inserted from either one of two points on the areola margin or one of two points at the inframammary fold in variable directions and planes to achieve a diffuse distribution

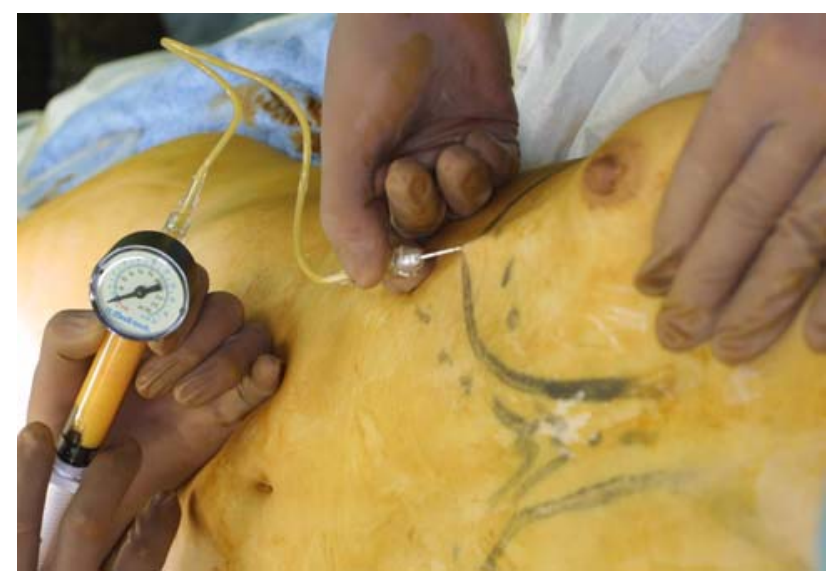

Fig. 3 A clinical view of injection. The injection needle is rigidly manipulated by an operator while an assistant rotates the plunger according to the operator's instruction. A high-pressure injection can be performed using a disposable syringe with a threaded plunger. A 150-mm-long, 18-gauge needle is connected to the syringe with a connecting tube threaded at both ends

without any palpable nodules at 6 months, and all the patients were satisfied with the resulting texture, softness, contour, and absence of foreign materials despite the limited size increase possible with autologous tissue-Cyst formation $(<12 \mathrm{~mm})$ was detected by magnetic resonance imaging (MRI) in two patients, and microcalcification was detected by mammogram in two patients at 24 months. In one of two patients in group B, fibrous breast tissue and fibrosis on the sternum were observed by computed tomography (CT) scan at 6 months, and the breasts were found to be harder than in other cases.

\section{Discussion}

Modifications of lipoinjection techniques to improve the survival rate for injected fat have been attempted. From these, it is well accepted that adipose tissue should be placed as small aliquots [3], preferably within an area 3 $\mathrm{mm}$ in diameter [1]. Because it takes a long time to perform ideally diffuse distribution of suctioned fat [3], we have used a disposable syringe with a threaded plunger and connections, a very long needle $(150 \mathrm{~mm})$, and an assistant to rotate the plunger. As a result, only 35 to $60 \mathrm{~min}$ are required for injection in both breasts. These devices are critical to performing large-volume lipoinjection safely and precisely in a short time.

In addition, the harvesting, preserving, and refining of graft materials also are important, as repeatedly indicated in the literature. We used a relatively large suction cannula, centrifuged the aspirated fat in some cases, and kept it cooled until transplantation. In this study, the clinical results (increase in breast circumference) appeared to be better in group $\mathrm{C}$ (using centrifuged fat) than in group $\mathrm{A}$ (using noncentrifuged fat), although quantitative measurement and statistical comparison were not done. In a previous study, we found that centrifugation of aspirated fat is substantially influential because centrifugation at $1,200 \mathrm{~g}$ decreases the fat volume by $30 \%$, damaging $12 \%$ of the adipocytes and $0 \%$ of the ASCs. This leads to the concentration of cell numbers per volume of adipocytes and ASCs by $25 \%$ and $43 \%$, respectively [7].

In addition, centrifugation may be especially beneficial in our treatment because water content in the graft material may disturb the adherence of ASCs to the adipose tissue and interfere with differentiation into expected lineages. Any ASCs floating in a solution, which is a nonphysiologic environment, may migrate over distances, penetrate into the lymphatic flow, and differentiate unexpectedly. We believe that such migration and altered cell differentiation caused the development of fibrotic tissue on the sternum of one patient in group B. Thus, we conclude that centrifuged fat combined with ASCs as cell pellets (group C procedure) was best among the three methods used in this study.

Although small cystic formation and microcalcification were detected in some cases, the microcalcification was easily distinguished from that associated with breast cancer, and the overall cosmetic results were generally satisfactory and encouraging. Almost all the patients were 
Fig. 4 Clinical views of a patient in group A (patient 1). Preoperative (left) and postoperative (right) views at 24 months. A 22-year-old woman underwent breast augmentation using cell-assisted lipotransfer (CAL) (290 $\mathrm{ml}$ in each breast), with satisfactory results at 24 months. Her breast circumference increased by 5 $\mathrm{cm}$, and her augmented breast mounds remained soft and natural appearing without injection scars or subcutaneous indurations
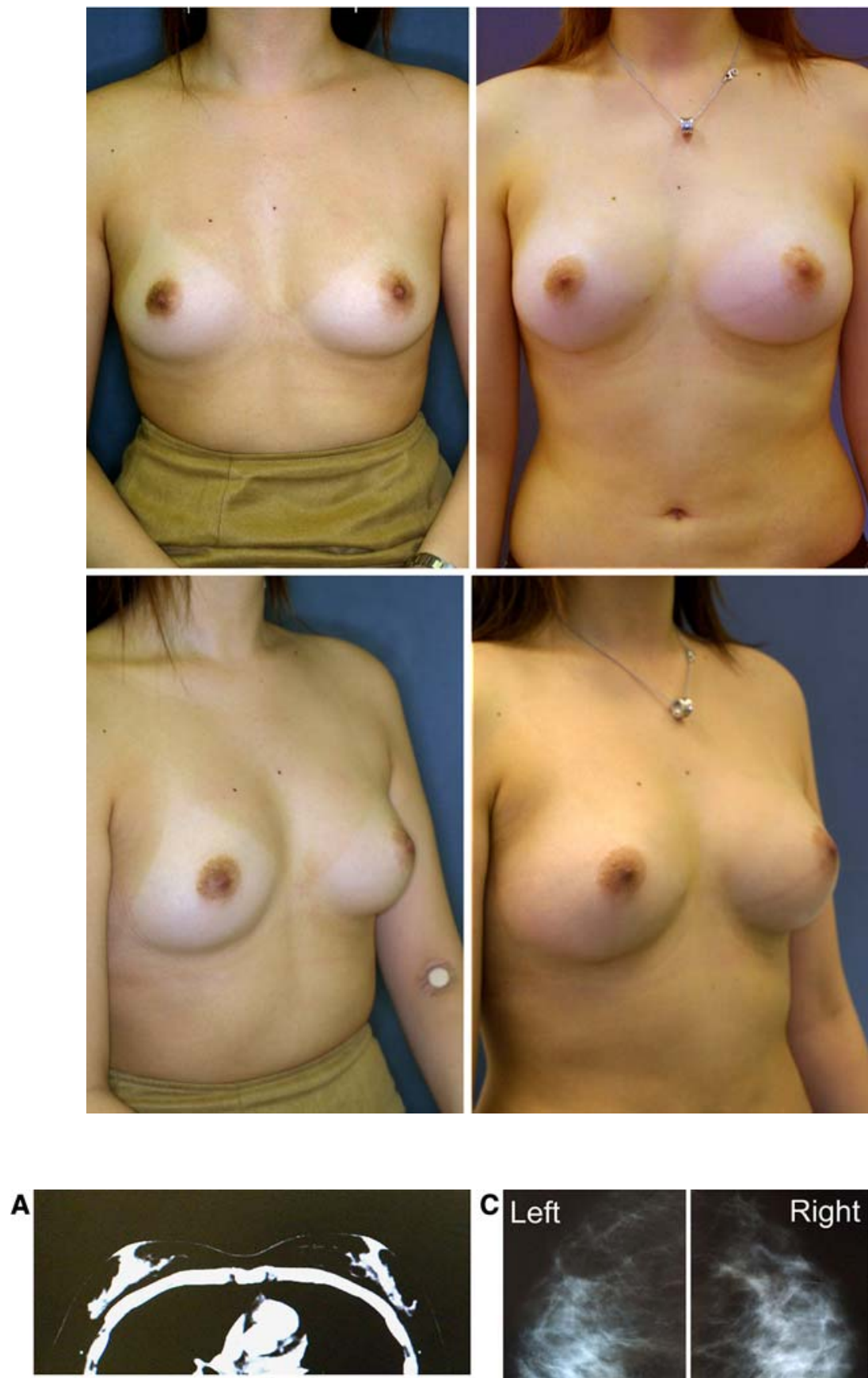

B
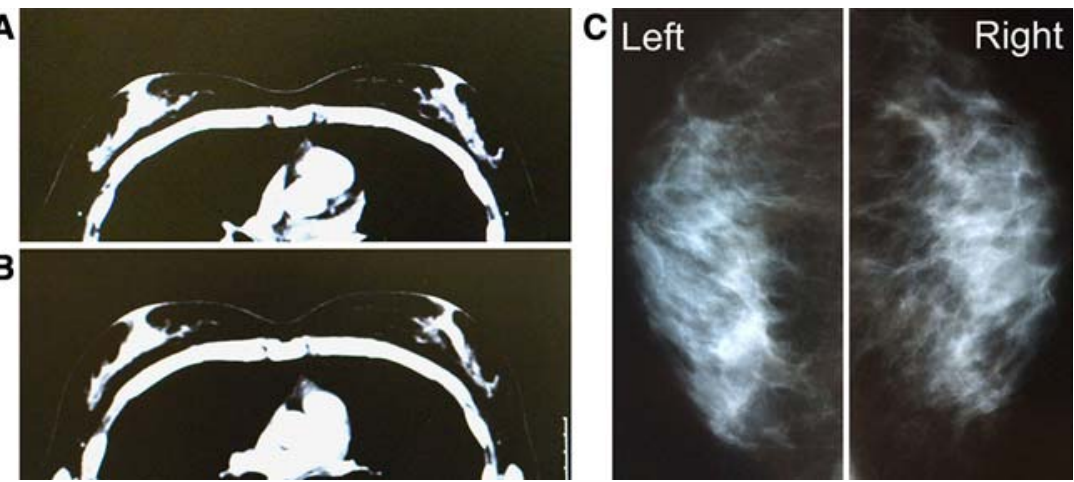

Fig. 5 Radiologic views showing the chest of patient 1 . (A) A preoperative computed tomography (CT) image in the horizontal plane of the nipples. (B) A horizontal image 12 months after surgery. Note that the adipose tissue is augmented both subcutaneously and under the mammary glands. $(\mathbf{C})$

Mammograms at 12 months show no calcification or other abnormal signs satisfied with their enlarged and soft breasts with a natural contour. Both CT scans and MRI showed that transplanted fat tissue survived and formed a significant thickness of the fatty layer not only subcutaneously on and around the mammary glands, but also between the mammary glands and the pectoralis muscles. Breast volume stabilized 2 to 3 months after transplantation.

Maximum breast augmentation using the described technique varied among the patients and appeared to be 100 to $200 \mathrm{ml}$. Although these volumes may be smaller 
Fig. 6 Clinical views of a patient in group C (patient 2). Preoperative (top) and postoperative (bottom) views at 12 months. A 32-year-old woman underwent breast augmentation with cell-assisted lipotransfer (CAL) $(280 \mathrm{ml}$ in each breast). Her breast circumference difference increased from $9 \mathrm{~cm}$ (baseline) to $14.5 \mathrm{~cm}$ (at 12 months). The breast mounds are soft and natural appearing with no visible injection scars
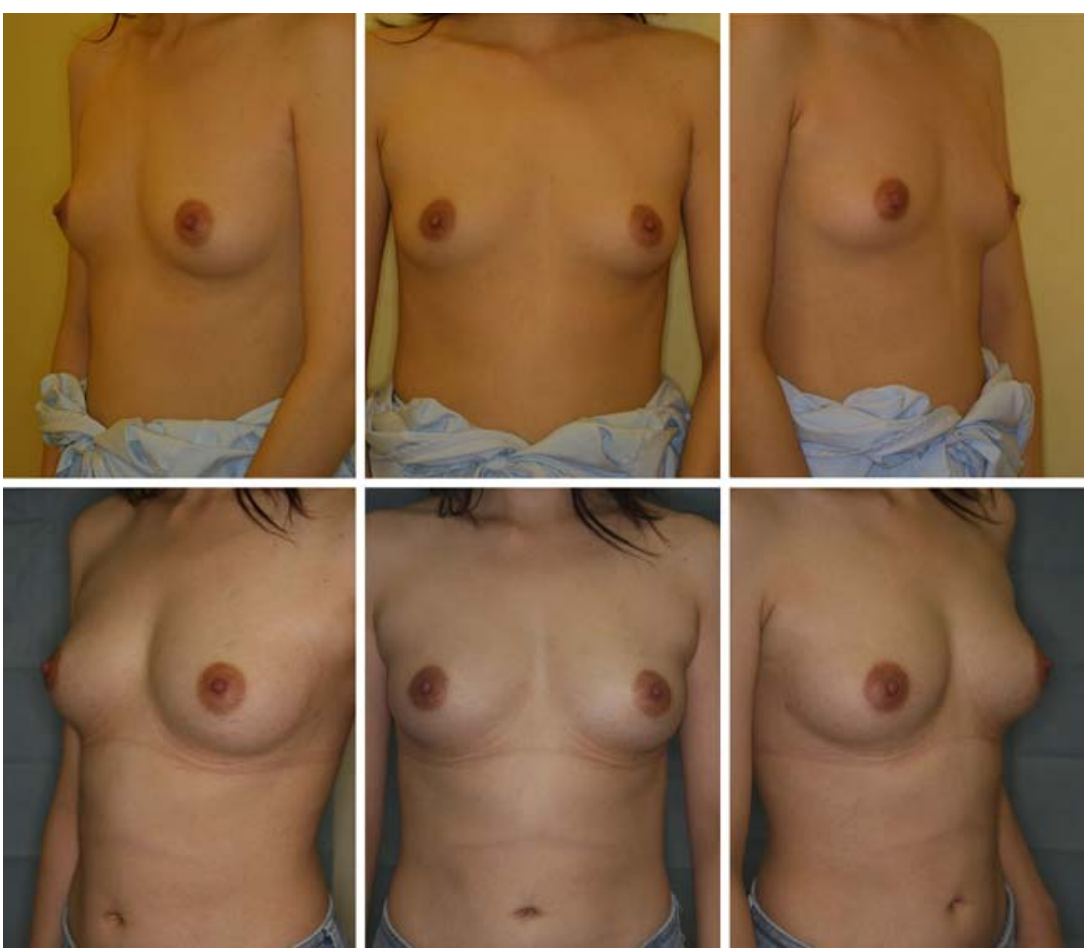

Fig. 7 Radiologic views showing the chest of patient 2 . (A) A preoperative computed tomography (CT) image in the horizontal plane at the level of the nipples. (B and $\mathbf{C}$ )

Horizontal images by magnetic resonance imaging (MRI) 12 months after surgery: (B) T1image; (C) T2-image. The adipose tissue is augmented around and under the mammary glands. A small cyst $(<10 \mathrm{~mm})$ appears in the fatty layer under the right mammary gland. (D) Mammograms at 12 months show no abnormal signs such as calcifications
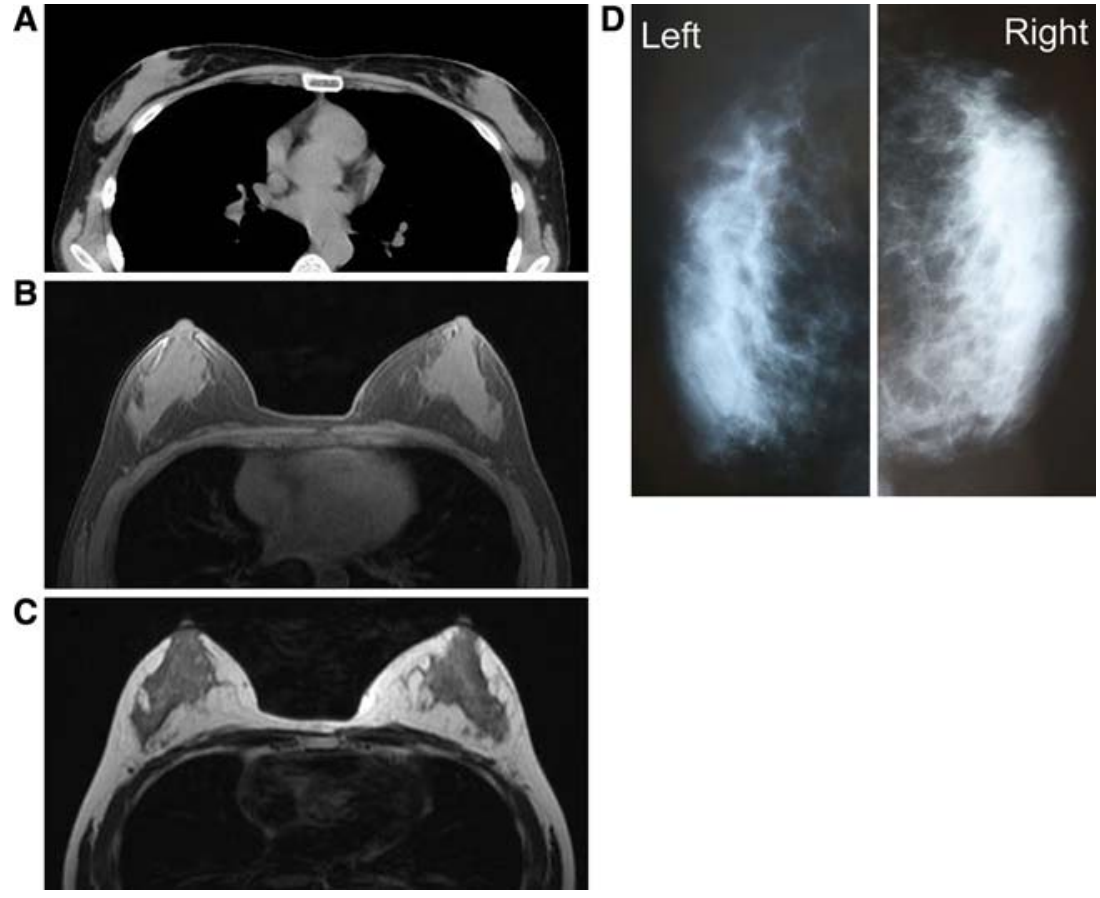

CAL. A 2- to 3-cm increase in breast circumference was common with the conventional procedure, compared with the 4- to $8-\mathrm{cm}$ increase seen in this trial of CAL, although the augmentation effect varied among patients. The measurement system we recently devised may help to quantify the difference in augmented volume in the future.

Adipose tissue contains not only adipogenic progenitor cells, but also multipotent stem cells, which can 
Fig. 8 Clinical views of a patient in group C (patient 3). Preoperative (top) and postoperative (bottom) views at 24 months. A 30-year-old woman underwent breast augmentation with cell-assisted lipotransfer (CAL) $(310 \mathrm{ml}$ in each breast). Her breasts were dramatically augmented with an increase in breast circumference difference by $8 \mathrm{~cm}$ at 24 months. The breast mounds were soft with no subcutaneous indurations. An original inframammary fold on the left breast is slightly visible, but injection scars are not visible
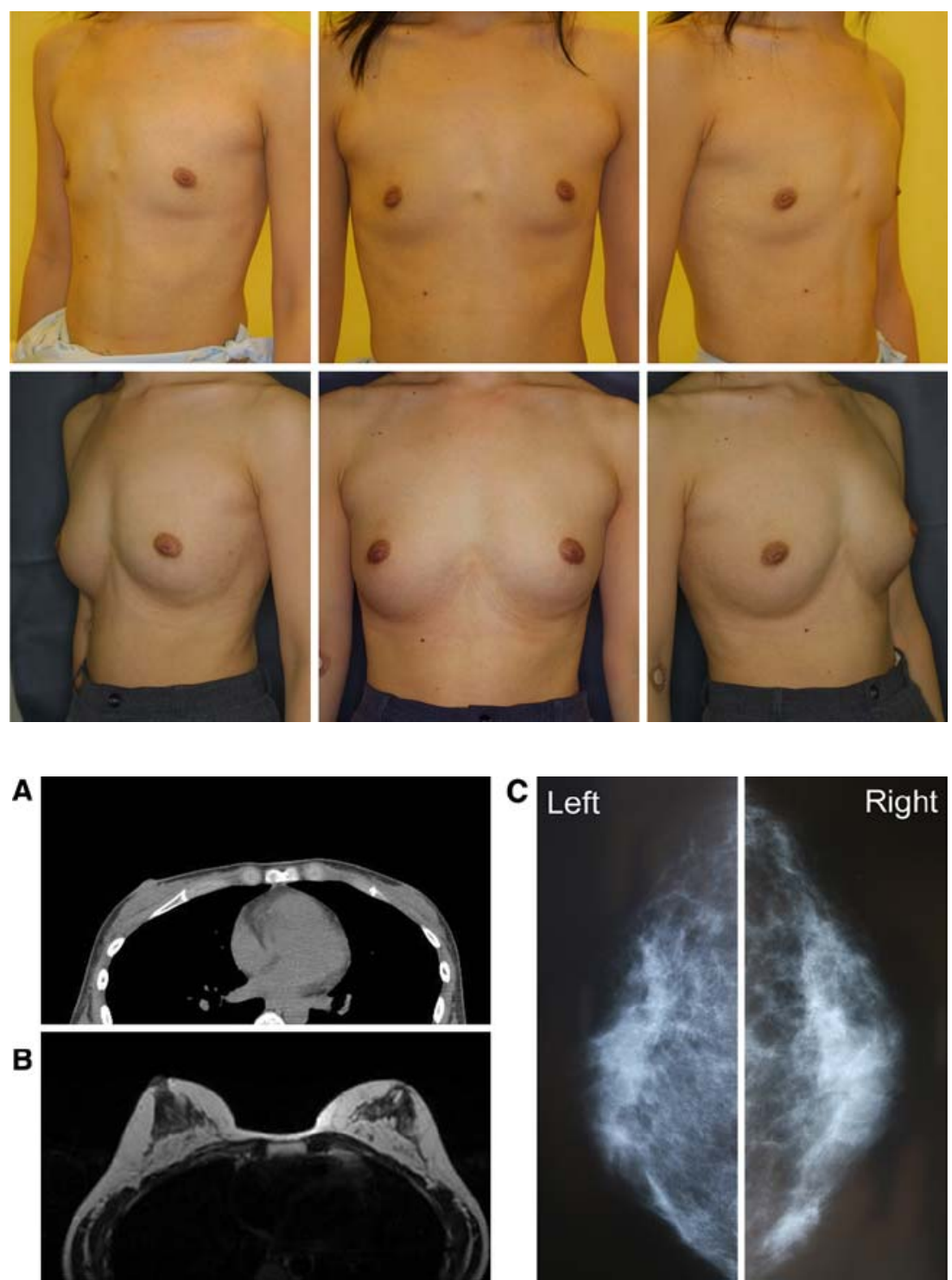

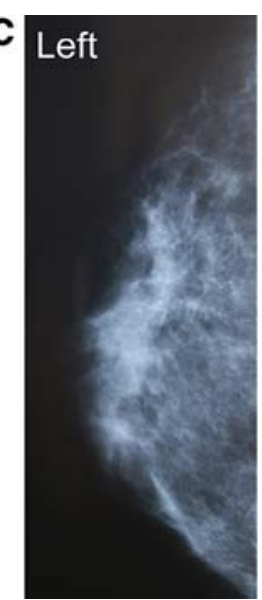

Fig. 9 Radiologic views of patient 3. (A) A preoperative computed tomography (CT) image in the horizontal plane at the level of the nipples. Only a very thin fatty layer is observed around the mammary glands.

(B) A horizontal magnetic resonance image (MRI) (T1 weighted) 24 months after surgery. Transplanted adipose tissues survived and formed thick layers around and under the mammary glands. $(\mathbf{C})$

Mammograms 24 months after surgery show no abnormal signs differentiate into fat, bone, cartilage, and types of tissue $[21,22]$. Suctioned fat appears to lose a significant number of these precursors during liposuction and the preparation processes as compared with nonsuctioned adipose tissue [9]. This relative deficiency of precursors may contribute to the low survival rate and long-term atrophy of transplanted lipoaspirates. In CAL, the deficit of ASCs was compensated by supplementing ASCs. To maximize the biologic function and avoid unexpected behavior of ASCs, it seems important to ensure adherence of supplemented ASCs to adipocytes or connective tissue.

With this novel treatment, ASCs have four possible roles, which were partly confirmed in preclinical studies [8, 9, 11]. First, ASCs can differentiate into adipocytes and contribute to the regeneration of adipose tissue. Second, ASCs can differentiate into endothelial cells and also probably into vascular mural cells $[8,10,12]$, resulting in the promotion of angiogenesis and graft survival. Third, ASCs are known to release angiogenic growth factors in response to hypoxia and other conditions [13], and these factors influence surrounding host tissue. In their final role, which may be the most influential, ASCs survive as original ASCs [9]. In the adipose tissue, ASCs reside between adipocytes or in the extracellular matrix, especially around vessels, and contribute to the turnover of adipose tissue, which is known to be very slow (2 years or more) [17]. However, adipose grafts probably turn over during the first 2 to 3 months after transplantation because they experience temporary ischemia followed by reperfusion injury. This turnover, the replacement process of the adipose tissue, is conducted by tissue-specific progenitor cells, which are ASCs. The relative deficiency of ASCs in aspirated fat may affect the replacement process and lead to postoperative 
atrophy of grafted fat, which is known to occur commonly during the first 6 months after lipoinjection.

The freshly isolated SVF used in CAL contains not only ASCs but also vascular endothelial cells, pericytes, blood cells (both white and red), and other cells, as previously described [20]. After transplantation, ASCs may interact with other cells such as vascular endothelial cells, and supplementation with the SVF may be superior to supplementation with ASCs alone in this treatment. However, further studies are needed to elucidate the synergistic effects of ASCs with other cells contained in the graft.

In this preliminary study, satisfactory clinical results were generally achieved without any major complications. Thus, we can conclude that CAL is sufficiently safe for continuation of the study, though controlled investigations and accumulated long-term results are needed to elucidate the overall safety and efficacy of the treatment. A variety of new innovations, including stem cell technology, may be developed and may contribute to the improvement of autologous tissue transplantation and regeneration. Further improvements of the technique may cause autologous tissue transfer to become the first choice for breast augmentation in the future.

\section{References}

1. Carpaneda CA, Ribeiro MT (1994) Percentage of graft viability versus injected volume in adipose autotransplants. Aesth Plast Surg 18:17-19

2. Coleman SR (2001) Structural fat grafts: The ideal filler? Clin Plast Surg 28:111-119

3. Coleman SR, Saboeiro AP (2007) Fat grafting to the breast revisited: Safety and efficacy. Plast Reconstr Surg 119:775-785

4. Ersek RA, Chang P, Salisbury MA (1998) Lipo layering of autologous fat: An improved technique with promising results. Plast Reconstr Surg 101:820-826

5. Fagrell D, Enestrom S, Berggren A, Kniola B (1996) Fat cylinder transplantation: An experimental comparative study of three different kinds of fat transplants. Plast Reconstr Surg 98:90-96

6. Har-Shai Y, Lindenbaum ES, Gamliel-Lazarovich A, Beach D, Hirshowitz B (1999) An integrated approach for increasing the survival of autologous fat grafts in the treatment of contour defects. Plast Reconstr Surg 104:945-954

7. Kurita M, Matsumoto D, Shigeura T, Sato K, Gonda K, Harii K, Yoshimura $\mathrm{K}$ : Influences of centrifugation on cells and tissues in liposuction aspirates: Optimized centrifugation for lipotransfer and cell isolation. Plast Reconstr Surg, (in press)

8. Masuda T, Furue M, Matsuda T (2004) Novel strategy for soft tissue augmentation based on transplantation of fragmented omentum and preadipocytes. Tissue Eng 10:1672-1683
9. Matsumoto D, Sato K, Gonda K, Takaki Y, Shigeura T, Sato T, Aiba-Kojima E, Iizuka F, Inoue K, Suga H, Yoshimura K (2006) Cell-assisted lipotransfer: Supportive use of human adiposederived cells for soft tissue augmentation with lipoinjection. Tissue Eng 12:3375-3382

10. Miranville A, Heeschen C, Sengenes C, Curat CA, Busse R, Bouloumie A (2004) Improvement of postnatal neovascularization by human adipose tissue-derived stem cells. Circulation 110:349-355

11. Moseley TA, Zhu M, Hedrick MH (2006) Adipose-derived stem and progenitor cells as fillers in plastic and reconstructive surgery. Plast Reconstr Surg 118(3 Suppl):121S-128S

12. Planat-Benard V, Silvestre JS, Cousin B, Andre M, Nibbelink M, Tamarat R, Clergue M, Manneville C, Saillan-Barreau C, Duriez M, Tedgui A, Levy B, Penicaud L, Casteilla L (2004) Plasticity of human adipose lineage cells toward endothelial cells: physiological and therapeutic perspectives. Circulation 109:656-663

13. Rehman J, Traktuev D, Li J, Merfeld-Clauss S, Temm-Grove CJ, Bovenkerk JE, Pell CL, Johnstone BH, Considine RV, March KL (2004) Secretion of angiogenic and antiapoptotic factors by human adipose stromal cells. Circulation 109:1292-1298

14. Shiffman MA, Mirrafati S (2001) Fat transfer techniques: The effect of harvest and transfer methods on adipocyte viability and review of the literature. Dermatol Surg 27:819-826

15. Spear SL, Wilson HB, Lockwood MD (2005) Fat injection to correct contour deformities in the reconstructed breast. Plast Reconstr Surg 116:1300-1305

16. Spear SL, Newman MK (2007) Discussion to "Fat grafting to the breast revisited: safety and efficacy." Plast Reconstr Surg 119:786-787

17. Strawford A, Antelo F, Christiansen M, Hellerstein MK (2004) Adipose tissue triglyceride turnover, de novo lipogenesis, and cell proliferation in humans measured with $2 \mathrm{H}_{2} \mathrm{O}$. Am J Physiol Endocrinol Metab 286:E577-E588

18. Ullmann Y, Hyams M, Ramon Y, Peled IJ, Leiderbaum ES (1998) Enhancing the survival of aspirated human fat injected into nude mice. Plast Reconstr Surg 101:1940-1944

19. Yoshimura K, Matsumoto D, Gonda K (2005) A clinical trial of soft tissue augmentation by lipoinjection with adipose-derived stromal cells (ASCs). Proceedings of the 3rd annual meeting of International Fat Applied Technology Society (IFATS), Charlotteville, Virginia, pp. 9-10

20. Yoshimura K, Shigeura T, Matsumoto D, Sato T, Takaki Y, Aiba-Kojima E, Sato K, Inoue K, Nagase T, Koshima I, Gonda K (2006) Characterization of freshly isolated and cultured cells derived from the fatty and fluid portions of liposuction aspirates. $\mathbf{J}$ Cell Physiol 208:64-76

21. Zuk PA, Zhu M, Ashjian P, De Ugarte DA, Huang JI, Mizuno H, Alfonso ZC, Fraser JK, Benhaim P, Hedrick MH (2002) Human adipose tissue is a source of multipotent stem cells. Mol Biol Cell 13:4279-4295

22. Zuk PA, Zhu M, Mizuno H, Huang J, Futrell JW, Katz AJ, Benhaim P, Lorenz HP, Hedrick MH (2001) Multilineage cells from human adipose tissue: Implications for cell-based therapies. Tissue Eng 7:211-228 